\title{
NOTAS SOBRE EL INSTITUTO \\ DE REFORMAS SOCIALES Y LAS TRES \\ HISTORIAS DE LA SOCIOLOGÍA \\ ESPAÑOLA $^{1}$
}

Juan Zarco

\begin{abstract}
RESUMEN
Por medio de tres definiciones de la palabra «historia» se presentan algunas de las obras fundamentales sobre la historia de la sociología española, algunos de los hechos que a ella pertenecen y algunos de los mitos y confusiones en torno a la misma. Todo ello sirve para introducir, contextualizar y evaluar el que aquí es considerado primer centro de estudio e investigación sociológica español: el Instituto de Reformas Sociales (1903-1924).
\end{abstract}

\section{INTRODUCCIÓN}

De las diez acepciones que tiene la palabra «historia» en el Diccionario de la Real Academia de la Lengua Española en su vigesimoprimera edición, de 1992, voy a tomar aquí tres que, por sus sentidos, explican y adelantan tanto mi interés en este trabajo como algunas de sus conclusiones. A ellas sumaré

${ }^{1}$ El contenido de esta nota de investigación procede de las investigaciones desarrolladas durante la realización de mi tesis doctoral, Presencia y desarrollo de la metodología cualitativa de investigación en el primer siglo de la sociología española (1875-1975), leída en la Facultad de Ciencias Políticas y Sociología de la Universidad Complutense de Madrid en mayo de 1998. Agradezco una vez más a quien fue director de la misma, Bernabé Sarabia, su incansable labor para hacerla llegar a buen puerto. 
una cuarta lección de diccionario, del diccionario de la historia de las palabras: el etimológico. Veamos cuáles:

La acepción primera de la palabra «historia» en el Diccionario de la RALE dice textualmente: «Narración y exposición de los acontecimientos pasados y dignos de memoria, sean públicos o privados». Dentro de esa acepción, sobre la sociología española es cierto que hay una - y varias — historias, que se han escrito numerosas reflexiones que narran y exponen acontecimientos pasados de nuestra sociología dignos de memoria. La cuarta acepción remite al "conjunto de los sucesos o hechos políticos, sociales, económicos, culturales, etc., de un pueblo o una nación». También en este sentido puede afirmarse hay un conjunto de hechos culturales pasados que podemos denominar y reconocer como sociología y que son de este pueblo o nación. La historia de la sociología española tiene, pues, también un objeto. Por fin, la última definición que tomo, que es la octava acepción del Diccionario, dice: "(fig. y fam.) Cuento, chisme, enredo». Pues también esta acepción nos sirve; como se señalará, sobre la sociología española hay cuentos, hay chismes y hay enredos.

Una cuarta lección de diccionario, en este caso del etimológico, nos recuerda que historia proviene del mismo término latino y éste del griego historía, que no es otra cosa que "búsqueda, averiguación». Por tanto, este texto es también en ese sentido una historia de la historia. Voy a decir algo de esas historias y de lo que de ellas toca a la sociología española hasta donde yo he hecho historía. Así, la historia uno, la historia cuatro y la historia ocho van a guiar esta presentación. Su fin es en parte instrumental; sólo presentando un panorama suficiente del contexto en que se enmarca este estudio - la sociología española, tanto por lo que de ella se ha narrado como por lo que en ella es objeto de historia y también por lo que en ella es cuento, chisme y enredo- puede situarse en todo su alcance el interés, valor y trascendencia de nuestro punto de atención último: el Instituto de Reformas Sociales (IRS).

\section{LAS TRES HISTORIAS DE LA SOCIOLOGÍA ESPAÑOLA}

La historia aquí llamada uno, la exposición de acontecimientos pasados dignos de memoria, tiene, por lo que se refiere a la sociología española, una tradición muy antigua. Es bien conocido, y en esta propia revista ha aparecido una reedición como texto clásico, que una de las primeras reflexiones en torno a la historia de la sociología española data de 1899. La firmó Adolfo Posada (1990) para el Boletín de la Institución Libre de Enseñanza, y, con ser antigua, no es siquiera la primera. Un año antes, Joaquín Costa escribía un epígrafe en su obra Colectivismo Agrario en España donde también aludía ya a la sociología española en términos históricos, si bien de manera tangencial. El trabajo de Posada es, sin embargo, el primero de entre aquellos que toman la historia de la sociología española en su conjunto como argumento sustantivo, ya sea en 
forma de libro, artículo de revista o epígrafe dentro de una obra mayor, y merece por ello una mención especial ${ }^{2}$.

Pero, además de estos trabajos que atienden de forma genérica y total la sociología española y su desarrollo, también son muchos los aspectos más o menos específicos que se han analizado de la misma. Así, existen trabajos sobre periodos concretos - aparte, claro está, de los que por su fecha de realización no podían llegar más allá de su propio límite temporal ${ }^{3}$ y también, aunque se trata de los aspectos más desatendidos, existen visiones sobre distintas especialidades $^{4}$, o sobre instituciones o elementos organizativos concretos 5 . Tam-

2 Cronológicamente a la reflexión de Posada siguió la voz «Spain and Portugal» para la Encyclopaedia of the Social Sciences, redactada por Fernando de los Ríos (1949) en 1930 para dar cuenta de la sociología en esos países; el apartado referente a «España y Portugal» del libro Historia del pensamiento social. Corrientes sociológicas en los distintos paises, de Barnes y Becker (1984), que se editó originalmente en inglés en 1938; "La sociología en España», capítulo firmado por Alfredo Mendizábal (1965) para el libro, editado por Gurvitch y Moore, Sociología del siglo XX, cuya primera edición vio la luz en 1947; "Sociología en España», de Enrique Gómez Arboleya (1958), quizá el más conocido de cuantos trabajos se han redactado sobre la materia, el cual, aunque reimpreso en numerosos lugares, fue firmado en 1956; la entrada «Sociología», redactada por Emilio Boix (1961) para la Enciclopedia Universal Espasa, que igualmente contiene un estudio del desarrollo de la sociología en España; el artículo "La sociología en España», de Antonio Perpiñá (1968); el libro Sociología o subversión, de Amando de Miguel (1972), que bajo tal título presenta una historia de la sociología española; el monográfico de la revista Documentación Social en su número 24, "Las ciencias sociales en España», a cargo de Vicente Sastre (1976); el igualmente monográfico, en esta ocasión de la publicación internacional Current Sociology, «Sociology in Spain», de Jesús M. de Miguel y Melissa Moyer (Miguel y Moyer, 1979); la obra Sociología en España, compilación de artículos a cargo de Salvador Giner y Luis Moreno (Giner y Moreno, 1990); Sociología, dirigido por Jesús Ibáñez (1992), dentro de la colección Las Ciencias Sociales en España, de la Editorial Complutense, y, por último, el epígrafe «Apuntes para una sociología de la sociología española», a cargo de Emilio Lamo de Espinosa (1994), contenido dentro del volumen, coescrito por ese autor junto a José María González García y Cristóbal Torres Albero, La sociología del conocimiento y de la ciencia. Hay que mencionar además que, aunque por el momento no se ha podido acceder a ella, existe, al menos, una tesis doctoral extranjera que, con el título The Development of Sociology in Spain, aborda la materia. Su autor fue John Lord, y la tesis —inédita, por desgracia - se leyó en la Universidad de Siracusa en 1921 (cf. en Barnes y Becker, 1984: 421, nota 1), lo que la sitúa cronológicamente entre las reflexiones de Posada y Fernando de los Ríos.

3 Se trata de trabajos como el de Luis Saavedra (1991), El pensamiento sociológico español, que se detiene en la Guerra Civil española; el de Diego Núñez (1973), que aborda solamente el período 1875 a 1914; aquel de José Jiménez Blanco (1985) que analiza «Diez años de sociología en España (1974-1984)»; el de Luis Rodríguez Zúñiga y Juan Salcedo (1991) a propósito de "Veinte años de sociología», o el de Xavier Marín y otros (1993) al hilo de los primeros veinte años de la revista Papers (1973-1993).

${ }^{4}$ Como la aportación de José Castillo (1973) sobre la sociología industrial, o la de Sarabia y Zarco (1997) acerca de la Metodología cualitativa en España.

5 Los trabajos de León Martín-Granizo (1947) o de Juan Ignacio Palacio (1988) sobre el Instituto de Reformas Sociales — sobre los que habremos de volver-; el firmado por la revista Perspectiva Social (1979) o el de Salvador Carrasco i Calvo (1980) sobre el Instituto Católico de Estudios Sociales de Barcelona (ICESB); el de Miguel Cancio (1995) sobre el Centro de Investigaciones Sociológicas (CIS); o el de Alfonso Ortí (1996) sobre CEISA-Escuela Crítica de Ciencias Sociales, por citar sólo algunos de ellos. 
bién es de señalar que se han publicado algunos repertorios bibliográficos de inmensa utilidad, como el contenido en Sociología española de los años setenta (VV.AA., 1971), realizado por Enrique Gómez Arboleya y que abarca hasta el año 1956; aquel que aparece en Sociology in Spain, a cargo de Jesús M. de Miguel y Melissa Moyer (1979); el del Seminario de Teoría Sociológica (1981) sobre los clásicos de la sociología en español, o los imprescindibles de Juan Díez Nicolás (1984 y 1989), todos ellos en cuanto se refiere a la sociología española en su conjunto ${ }^{6}$. Asimismo, existen monografías y trabajos sobre distintos autores clásicos entre nosotros, como los publicados sobre Joaquín Costa $^{7}$, Manuel Sales y Ferré ${ }^{8}$, Gumersindo de Azcárate ${ }^{9}$, Severino Aznar ${ }^{10}$, Adolfo Posada ${ }^{11}$, Enrique Gómez Arboleya ${ }^{12}$, o los más recientes en torno a figuras tan distantes como la de Jesús Ibáñez (p. ej. Álvarez-Uría, 1997) y Cánovas del Castillo, con la aportación de Salustiano del Campo (1997) al libro homenaje Antonio Cánovas del Castillo. Homenaje y memoria de la Real Academia de Ciencias Morales y Politicas (1897-1997), de reciente aparición, y donde se introducen muy interesantes reflexiones sobre el doble origen de la sociología española y el papel desempeñado por Cánovas en ella.

Por desgracia, no hay abundancia de documentos personales o escritos en primera persona. Se han publicado, a menudo de forma póstuma, algunas memorias, de entre las que son un ejemplo las de Adolfo Posada, del que se ha publicado una parte de su diario (cf. Sánchez Agesta, 1983); de algunos autores se han conocido también epistolarios de mucho interés (de Francisco Giner de los Ríos o de Gumersindo de Azcárate, por ejemplo); pero no hay, como sí entre sociólogos de otros lugares, historias de vida o autobiografías intelectuales, y cuando, como en el caso de Juan José Linz (1994 y 1997) o de Salvador Giner (1984), se ha tomado la pluma en primera persona se ha primado en exceso el relato del trabajo intelectual sobre otros aspectos vitales que, sin duda, imbricados con aquél, contribuirían a enriquecerlo. Quizá sean una excepción la autobiográfica "Autopercepción intelectual de un proceso histórico», de Jesús Ibáñez (1990), donde sí aparecen varios datos vitales que colabo-

6 También son de utilidad los de José Cazorla (1967) o Guy Hermet (1968/1969), ambos sobre sociología empírica española.

${ }^{7}$ Las reflexiones de Luis Sarries Sanz (1986) o Elías Díaz (1996) y, sobre todo, las de Alfonso Ortí, con su célebre y precursor prólogo a Colectivismo agrario...

8 Trabajos de Tomás Maestre (1962), Manuel Núñez Encabo (1976), Rafael Jerez Mir (1981), Enrique Martín López (1992) o José Enrique Rodríguez Ibáñez (1996) sobre Manuel Sales y Ferré

9 Sobre el pensamiento político de Gumersindo de Azcárate es imprescindible la mención al trabajo de José Ramón Torregrosa (1964). También las reflexiones de Elías Díaz (1979) o la muy documentada biografía de Pablo de Azcárate (1969) sobre el mismo autor.

${ }^{10}$ La tesis de María Mercedes López Coira (1986).

${ }^{11}$ Los trabajos de Francisco Laporta (1974) o Luis Sánchez Agesta (1983).

12 De imprescindible mención es el trabajo en el que, con la colaboración de varios autores (VV.AA., 1988), se le rindió homenaje desde la Universidad de Granada, o la aportación de Jiménez Blanco (1984) sobre el mismo autor. 
ran a la comprensión de su autor; o la biografía intelectual de Esteban Pinilla de las Heras contenida en el libro de J. F. Marsal (1979) Pensar bajo el franquismo, bajo el seudónimo de «Sartorius»; o la más reciente del mismo Pinilla de las Heras en una edición póstuma del Centro de Investigaciones Sociológicas a cargo de Jesús M. de Miguel (Pinilla de las Heras, 1996). El caso de Francisco Ayala (1984), con sus Recuerdos y olvidos, quizá las únicas memorias extensas de un sociólogo español, tampoco nos resarce totalmente pues en ellas parece recordarse su carrera literaria — valiosísima sin duda- y olvidarse su papel en la sociología, si se permite el juego de palabras. También algunas «escuelas»o líneas de pensamiento han sido estudiadas: el krausismo por Elías Díaz (1989) o el positivismo por Diego Núñez (1987) son sólo dos muestras de ello.

Los contenidos de todos estos trabajos, sin excepción, son de algún modo los aludidos en la definición cuarta que hemos presentado de historia — «conjunto de los sucesos o hechos políticos, sociales, económicos, culturales, etc., de un pueblo o una nación»- . Todas estas obras hacen historia de la sociología española y, por tanto, recogen en sus contenidos hechos históricos de la disciplina, pues tal es su objeto de reflexión. Es evidente que las aquí llamadas historia uno e historia cuatro son dos dimensiones del mismo fenómeno, vale decir continente y contenido. Algunos de los trabajos señalados hacen alusión ya desde el propio título a aspectos concretos, a personas e instituciones; a elementos, en fin, de la historia de nuestra sociología. Así, la fecha de 1899 es señalada como la que vio nacer la primera cátedra de sociología de la universidad española ${ }^{13}$; las figuras del propio Manuel Sales y Ferré, Adolfo Posada o Gumersindo de Azcárate, como los tres representantes más destacados de la primera época; el Instituto de Estudios Políticos, el Instituto Balmes de Sociología o el Instituto de la Opinión Pública, como instituciones relevantes para su posterior desarrollo; las empresas privadas de investigación como DATA, ECO o Iberométrica, como lugares de formación y entrenamiento de sociólogos; la cátedra de Gómez Arboleya en la Universidad de Madrid y su trascendencia, el llamado grupo de Granada, o las distintas iniciativas de institucionalización en Cataluña; la salida a Estados Unidos de sociólogos españoles becados a partir de los años cincuenta; o, en fin, la creación, entre los años 1972 y 1974, de la primera facultad de sociología oficial y pública, son todos ellos elementos presentes en las distintas narraciones sobre la sociología española ${ }^{14}$. En verdad, y a la vista de la abrumadora cantidad de escritos existente, de la que los señalados son sólo una muestra, puede decirse que casi cualquiera capaz de

${ }_{13}$ Hecho que durante este año viene conmemorándose desde distintas instituciones.

${ }^{14}$ Los límites marcados por el contexto de esta nota sólo posibilitan nombrar dichas narraciones, constituyendose así en lo que puede parecer mera enumeración, y ni siquiera exhaustiva. Quien esté interesado en un análisis de cierta profundidad sobre las distintas etapas, autores y contribuciones de la sociología española que aquí se mencionan puede remitirse al libro Metodología cualitativa en España (Sarabia y Zarco, 1997), donde, al hilo de esa perspectiva de investigación, se realiza un análisis de dichas contribuciones, que aquí, por desgracia, en la mayoría de los casos sólo podrán ser mencionadas. 
mover pluma en la sociología española ha escrito algo sobre precisamente la sociología española. Pudiera considerarse, por tanto, que la práctica totalidad de los aspectos de interés de nuestra sociología pasada han sido, en mayor o menor medida, tratados por los autores señalados. Esta afirmación es sin duda en parte verdadera, pero, como veremos, existen excepciones.

La octava definición del Diccionario de la RALE, la que recoge el término en uno de sus sentidos figurados, nos advierte, sin embargo, de que bajo la palabra historia pueden presentarse narraciones inexactas, cuentos — «breve narración de sucesos ficticios y de carácter sencillo, hecha con fines morales o recreativos»—, es decir, simplificaciones; también chismes — «noticia verdadera o falsa, o comentario con que generalmente se pretende indisponer a unas personas con otras...»- que se repiten como consignas; $y$, en fin enredos, malentendidos o confusiones. Por supuesto que no soy el primero en advertirlo, ni siquiera por escrito. Quizá una de las más agudas reflexiones en este sentido fuera la que realizó José Vidal Beneyto hace ya más de un cuarto de siglo, cuando se refirió a una de las historias de la sociología mencionadas, recién aparecida en aquella fecha, en estos términos cinematográficos: «... asistimos a la enconada lucha que sostiene el bueno o el chico (que así se llamaba en mi pueblo) o el protagonista - la sociología empírica- contra los indios por una parte - representados por la Filosofía Social y la Sociología católica- llenos de buena voluntad pero torpones, anticuados, tercos; y los bandidos por otra, encarnados por la Sociología crítica, radical, marxista, marxiana, etc., irremediablemente perdidos en la vaguedad, el sometimiento a un credo, la cobardía, el dogmatismo, la ambivalencia, etc.» (Vidal Beneyto, 1973: 261).

Aquí, como entonces, los cuentistas son lo de menos, que cada palo aguante su vela, pero lo que sí interesa es señalar la existencia de estos cuentos (películas en el caso de la ironía de Vidal Beneyto), de estos chismes y estos enredos, porque lo que importa es que si el profesor Vidal Beneyto nos está indicando con su segunda versión que no hay unos buenos, unos bandidos y unos indios es porque el creérnoslo nos oculta los verdaderos acontecimientos o, al menos, otra posible interpretación de los mismos.

La caricatura de Vidal Beneyto es sólo el ejemplo; el lector, entre todas las historias uno que pueda leer o haber leído, y todos los hechos de la historia cuatro que recuerde o conozca, podrá sin duda precisar muchas historias ocho de nuestra sociología. Mi objetivo aquí, sin embargo, es detenerme solamente en una institución concreta, unas personas, y unos elementos a ella ligados que colorean, creo que más precisamente de lo que hasta ahora lo ha sido, un período de nuestra historia rico, brillante $y$, hasta donde he podido averiguar, poco o nada difundido.

No se piense que los acontecimientos a los que remitía la crítica transcrita, que hacía referencia a un momento concreto de la historia de la sociología española situado entre mediados de los años cincuenta y mediados de los sesenta aproximadamente, y el Instituto de Reformas Sociales, que funcionó desde 1903 a 1924 y que enseguida será destacado, quedan, por distantes, 
inconexos. Denunciar, por inexacto, el papel de héroe y protagonista absoluto de la así llamada en la época "sociología empírica» en los años cincuenta/ sesenta, de una parte, y enfatizar el interés y trascendencia del Instituto de Reformas Sociales para la sociología española, de otra, remiten ambos argumentos directa o indirectamente a uno y mismo «acontecimiento digno de memoria» concreto de la historia de la sociología española. Tal es, ni más ni menos, el propio nacimiento de la sociología española. Como acabo de afirmar y puede suponerse, no es el único de los elementos de nuestra historia objeto de cuentos, chismes y enredos, pero puede comprenderse también que, por su temática, se trata de uno de los de más calado. Efectivamente, porque en torno a cuándo, cómo, con quiénes y por qué nació la sociología española se concentran muchos de los cuentos - sobre todo por lo que en ello se ha simplificado-, muchos de los enredos y también los chismes, pues con ellos se consigue indisponer a unos con otros. $\mathrm{Y}$ en torno a la fecha, acontecimiento, personas o institución que puedan considerarse como los fundacionales de la sociología española gira el tema de fondo de esta reflexión.

Si se me permite tomar prestada como metáfora la fábula de Borges sobre el mapa tan realista que finalmente cubrió y suplantó al territorio, metáfora que tan buen resultado dio a Baudrillard (1984: 9 y ss.) en Cultura $y$ Simulacro; para nuestro objeto de estudio puede afirmarse que todas las aportaciones señaladas constituyen un mapa muy completo del territorio que estudiamos, la sociología española pasada. Sin embargo, si superponemos el mapa al territorio, podemos observar que algunas orografías están detalladas milimétricamente mientras otras se han cartografiado, diríamos, con brochazos imprecisos. Hay elementos que no han quedado bien reproducidos en el mapa, los lugares que representan son ficticios, no existen, o no con esa fisonomía; o, por el contrario, elementos que de hecho existen en el territorio - existieron en nuestra sociología - han sido, más que borrados del mapa, nunca reflejados en él. Algo he mencionado de estos últimos, que representan en el mapa historias ocho mal dibujadas, no importa por qué cartógrafos, y aunque sí interesaría dilucidar si por miopía o por cuentismo interesado - pues el juicio sería bien distinto-, no voy aquí a entrar en ello. El contenido del epígrafe siguiente no responde tanto a malas representaciones del mapa - que en el fondo también lo son pues lo vuelven incompleto- como a una de las ausencias de detalle, a una de esas zonas del territorio de las que el mapa nos ofrece sólo unos leves contornos, privándonos de sus rasgos quizá más esenciales. El conjunto del mapa con estas zonas borrosas nos remite a una realidad desfigurada. Una de esas zonas borrosas es, según me parece, el Instituto de Reformas Sociales, y el intento de clarificar sus rasgos en este nuevo mapa — pues no es otra cosa esta reflexión- quizá contribuya a la mejor caracterización - $-\mathrm{y}$ conocimiento- del territorio al que remite. 


\section{EL INSTITUTO DE REFORMAS SOCIALES}

\section{a) Introducción, genealogía y objetivos del IRS}

La mayoría de los autores que desde la sociología atienden a las fechas del primer cuarto del siglo XX coinciden al destacar la labor desempeñada en la época por el Instituto de Reformas Sociales (cf. Gómez Arboleya, 1958, o Boix, 1961). Para Lamo de Espinosa, por ejemplo, se trataba del único núcleo institucional que "continuaba ocupándose del problema social y produciendo notables investigaciones empíricas» (Lamo et al., 1994: 595). Boix lo ha descrito como una «zona de diálogo, de coordinación e incluso de colaboración entre personalidades de distintas tendencias en el ámbito de los estudios sociológicos y también de la legislación y la acción» (Boix, 1961: 1383). Y una similar interpretación ofrece Mendizábal, quien afirma que: «Tuvo, además, el mérito, poco común en ese país [España], de crear un ambiente propicio a la cooperación de las distintas tendencias y escuelas. Las más características de éstas fueron adecuadamente representadas en el Instituto, a saber: a) la tendencia ético-organicista basada en el krausismo [...] b) el socialismo en sus dos aspectos, intelectual y de organización obrera; c) el catolicismo social, inspirado en las doctrinas eclesiásticas [...]" (Mendizábal, 1965: 349). Aunque existen otras alusiones, a menudo incidenciales, y quizá simplificando al máximo, digamos que fuera de estos autores, desde la sociología, poco más aparece en las crónicas. No ocurre lo mismo desde otras perspectivas, como la históricojurídica (Palacio Morena, 1988) o la institucional (Instituto de Reformas Sociales, 1919, o Martín Granizo, 1947), desde las que se le ha prestado mayor atención o, al menos, de mayor profundidad ${ }^{15}$.

Repasemos en primer lugar la genealogía del Instituto de Reformas Sociales. En 1855 —según se afirma en El Instituto de Reformas Sociales (1919), publicación editada por el propio Instituto para su difusión y conocimiento público - puede buscarse su primera ascendencia o linaje, ya que en esa fecha se crea el primer "Centro para el estudio de los problemas sociales» por el que, por aquel entonces, era el presidente del Congreso: don Pascual Madoz. Esa iniciativa se va perfilando y concretando en los años siguientes, siendo en 1883 cuando "un Real decreto del señor Moret" promulga la "Comisión para el estudio de las cuestiones que directamente interesan a la mejora o bienestar de las clases obreras, tanto industriales como agricolas, y que afectan a las relaciones entre el capital y el trabajo" (citado en El Instituto de Reformas Sociales, 1919: 3). El más directo antecedente del Instituto es, sin embargo, la Comisión de Reformas Sociales, creada por Real decreto de marzo de 1890, como evolución y formalización de los organismos predecesores ya mencionados, y a la que en seguida

15 El trabajo de Palacio Morena contiene el análisis más profundo de los que he podido encontrar y, pese a proceder de una perspectiva más jurídica que sociológica, supone el mejor instrumento para el conocimiento de la Institución por parte de sociólogos y politólogos. 
volveremos. Así, y por fin, «la idea generadora del Instituto fue recogida y amplificada con considerable éxito por el Sr. Silvela en el Real decreto de 23 de abril de 1903, al crear el Instituto de Reformas Sociales, idea desarrollada con todo detalle en el Reglamento [...] La creación del Instituto equivale, en el proceso de estos organismos del Trabajo y de la Reforma social, a la de las Oficinas o Departamentos del trabajo en casi todos los países; es el momento en que el Estado, dándose plena cuenta de la complejidad del problema social y de la necesidad de realizar una intervención prudente, preparada, organiza el servicio público indispensable: 1. ${ }^{\circ}$ Para realizar las informaciones necesarias del movimiento social, tanto en el propio país como fuera de él [...] 2. ${ }^{\circ}$ Para procurar al Gobierno las consultas y el consejo en todo lo que se refiere al ejercicio de la función interventora; 3. ${ }^{\circ}$ Para hacer efectiva la aplicación de las Leyes tutelares del obrero mediante la inspección» (op. cit.: 4-5). Vemos, pues, que, ante la complejidad del problema social, estudio, comparación con el extranjero, asesoramiento al Gobierno e inspección para la aplicación de leyes tutelares fueron los elemento para los que se concibió el Instituto de Reformas Sociales.

El Instituto de Reformas Sociales tuvo un antecesor directo, la Comisión de Reformas Sociales, que durante veinte años se ocupó, con menos medios y menor autonomía, de los asuntos en los que, como transformación de la misma, pasaría a ocuparse el Instituto. De la Comisión existe un muy documentado trabajo de María Dolores de la Calle (1989) titulado La Comisión de Reformas Sociales (1883-1903). Allí, su autora afirma que: «El método de trabajo de la C. R. S. se sustenta en los avances científicos de la época. La importancia que va adquiriendo la sociología impulsa los estudios de la realidad social y condiciones de la vida obrera» (Calle, 1989: 14). Asimismo, señalemos que el Ministerio de Trabajo en 1985 publicó una edición facsímil en cinco volúmenes de la publicación oficial de la Comisión que, bajo el título Reformas sociales: información oral y escrita publicada de 1889 a 1893, recoge, además de aquélla, un estudio introductorio del historiador y sociólogo Santiago Castillo de gran interés (Comisión de Reformas Sociales, 1985).

En la Comisión participaron ya algunos de los nombres que suelen ser aludidos como los de los representantes de la primera sociología española, pero de entre ellos vamos a tomar aquí la referencia a Gumersindo de Azcárate, pues un trabajo suyo va a servirnos para introducir cierta «manera» o estilo — si es que quizá no haya de llamarse aún metodología- de investigación. Desde luego, sí parece una de las primeras muestras de ensayo de una técnica de investigación sociológica en la sociología española: la técnica de encuesta. Así, en 1884, en una de las primeras reuniones de la recientemente creada Comisión, se encarga a Gumersindo de Azcárate (secretario de la misma) la redacción de un cuestionario que ha de proveer de los datos a la «Comisión para el estudio de las cuestiones sociales que interesan a la mejora o bienestar de las clases obreras, tanto agrícolas como industriales, y que afectan a las relaciones entre el capital y el trabajo» (cf. en Calle, 1989: 330 y ss., apéndice II). El pro- 
pio título de esa Comisión sería objeto por sí mismo de un análisis de contenido que informaría sobre el espíritu de la Comisión de Reformas Sociales. Otro tanto podríamos afirmar del cuestionario redactado por Azcárate, el cual constó de 32 apartados, los cuales generaron un total de 223 preguntas. Aunque no podemos acometer aquí esa tarea, sí podemos, sin embargo, presentar algunas consideraciones que parecen de interés.

Efectivamente, el solicitar la redacción de un cuestionario parece una tentativa de generar información de tipo estadístico de forma específica para el conocimiento de un aspecto concreto, en este caso de un sector de la realidad social, "las clases obreras, tanto agrícolas como industriales». La redacción del cuestionario de Azcárate nos haría hoy día calificarlo, sin dudar, de cuestionario de "preguntas abiertas». Primaba, sin duda, la adquisición de la mayor cantidad posible de información sobre posibles criterios de codificación que aún no estaban en el horizonte. El cuestionario, en cualquier caso, viene reproducido en el ya citado trabajo (Calle, 1989: 330 y ss.) y a él remitimos. Señalemos que, aunque desde el punto de vista de la técnica de encuesta presenta elementos de dificultad (dimensión extensísima, preguntas de difícil respuesta, sesgos ideológicos, solapamiento de contenidos, etc.), representa un trabajo pionero en la sociología del que ha parecido oportuno hacerse eco, máxime por tratarse de un hecho tan poco aludido en la literatura sobre la materia. Sorprende a este respecto que no haya sido recogido, por ejemplo, por Amando de Miguel (1987) en su trabajo "Las primera encuestas en España», publicado en la obra homenaje a Francisco Murillo Ferrol, donde el primer autor al que menciona es a Joaquín Costa (por su trabajo de 1903) y no vuelve a detenerse hasta los años cincuenta.

Pero aunque la Comisión, en cuanto antecedente directo del Instituto, interesa pues, como se ha señalado, ya en ella pueden encontrarse signos de la alborada de nuestra sociología, es en el Instituto donde con más sorprendente claridad va a aparecer la huella de una primera sociología española robusta. Ella quedará plasmada o encarnada en la conjunción manifiesta de al menos dos tipos de elementos. Por una parte, las fuentes documentales bibliográficas con las que contó; por otra, las investigaciones - como veremos, caracterizadas por sus propios autores como de investigación sociológica- que se realizaron; a ambos elementos puede sumarse la labor de difusión tanto de los contenidos de las primeras como de las conclusiones de las segundas. Estos elementos que a continuación se desarrollarán le confieren por derecho propio el grado de primer centro de estudio, investigación y difusión de la sociología española.

\section{b) Las publicaciones en $y \operatorname{del} I R S$}

A iniciativa de Adolfo Posada se publicaron por el Instituto, desde el año 1907 y hasta 1913, seis volúmenes en forma de anuarios, cuyo cometido era dejar constancia y hacer pública la actividad de la institución. Voy a tomar esas 
obras, de incalculable valor documental, en uno de sus apartados, el que da cuenta de las publicaciones periódicas recibidas por el Instituto en el área de la sociología $a^{16}$. Lo que Posada publica en realidad es una relación (ordenada por materias) de los artículos aparecidos en las revistas que el Instituto de Reformas Sociales recibía periódicamente. El propio Posada explica en el prólogo del texto aludido que algunas de las publicaciones son compradas por suscripción y algunas otras - las más de ellas- intercambiadas por la publicación del Instituto, el Boletín del Instituto de Reformas Sociales, procedimiento aún frecuente entre bibliotecas de instituciones o facultades. De los anuarios referentes a las publicaciones recibidas entre los años 1906 y 1912 podemos extraer al menos tres aspectos destacables: por una parte, las revistas que el IRS recibía; por otra, los autores que en ese sexenio y en dichas publicaciones colaboraron con sus trabajos; y, finalmente, las temáticas o materias que en esas fechas eran objeto de estudio o interés en la sociología internacional.

En cuanto a las publicaciones periódicas que el Instituto recibía, y entre todas ellas, pueden mencionarse por su significación el American Journal of Sociology, Rivista Italiana de Sociologia, Rivista Internazionali di Sciencia Sociali, Revue Internationale de Sociologie, Archiv für Socialwissenschaf, The Sociological Review y Revue de Psychologie Sociale. Como puede observarse, las sociologías de habla anglosajona, italiana, alemana y francesa quedan representadas. Pero, más allá de las publicaciones, que sin embargo no deben menospreciarse pues, como se ha apuntado, no se trata de números sueltos o individuales, sino de colecciones completas, con la riqueza de contenidos que el carácter temporal les imprime, y en las cuales sin duda era posible descubrir líneas de pensamiento y de evolución de tendencias, podemos también apuntar algunos autores y temáticas, pues son en definitiva los autores, y por tanto los contenidos que los primeros sociólogos españoles pudieron conocer a través de las revistas recibidas en el Instituto, lo que más importa determinar.

Cronológicamente, el primer año que Posada recoge es el 1906, y esta fecha nos introduce en el primer elemento que interesa destacar. Efectivamente, 1906 es la fecha centenario del nacimiento de Frédéric Le Play, hecho que no pasa desapercibido en la sociología internacional — particularmente para la francesa- y que es conmemorado en varios trabajos de ese año. Esta circunstancia queda reflejada en La Reforme Sociale — publicación que también recibía el IRS-, revista que dedica, a lo largo del año 1906, cuatro artículos, en números distintos de su publicación, al análisis de la sociología de Le Play. Resulta quizá más significativo destacar los numerosos trabajos de Simmel que en esos años aparecían en revistas alemanas como el Archiv für Sozialwissenschaft, ya mencionada, o en Jahrbüch für Gesetzgebung, ambos trabajos fechados

${ }_{16}$ Todas las referencias que a continuación se señalarán han sido tomadas directamente de las publicaciones originales del Instituto, que son de consulta pública en la biblioteca del Ministerio de Trabajo y Asuntos Sociales. Dicha biblioteca contiene tanto los catálogos a los que se señala (de los años 1906 al 1912) como las obras en ellos recogidas. 
en 1907, pero también francesas como La Vie Contemporaine y norteamericanas como The American Journal of Sociology, donde la obra de Simmel aparecía traducida al francés y el inglés. Trabajos sobre el problema de la sociología, la sociología del secreto o la psicología de la hostilidad son algunas de las aportaciones de Simmel. Otro tanto podemos afirmar de contribuciones, menos numerosas pero igualmente sustantivas, del propio Max Weber, quien, como es sabido, desde esos años en que publicaba La ética protestante y el espiritu del capitalismo (1904 y 1905) y hasta su muerte en 1920 tuvo uno de los períodos más creativos de su carrera. De entre los muchos otros sociólogos que aparecen con artículos en esas publicaciones podemos igualmente destacar a los pioneros norteamericanos, de entre los que resaltan trabajos firmados por Alvion Small o Lester Ward.

En cuanto a las áreas temáticas que son paradigma de la sociología de la época pueden destacarse los Social Surveys y, progresivamente, los estudios de minorías étnicas y de otras marginalidades, precedentes directos de los estudios desarrollados en los años siguientes en la Universidad de Chicago. Así, trabajos como los de Riley, "Sociological and Social Surveys»; los de Cohen sobre la comunidad judía, o aquellos con el interés centrado en The American Negro - temática central en muchas investigaciones norteamericanas y que, más de treinta años después, el sociólogo, economista y premio Nobel sueco Gunnard Myrdal inmortalizaría como "el dilema americano»-, iban apareciendo en las revistas científico-sociales norteamericanas.

Éstos son algunos de los datos; el análisis de los mismos está, obviamente, sujeto a interpretación. Sin embargo, son indicativos de uno de los varios elementos que han de considerarse para evaluar el grado de información de un grupo de intelectuales centrados en torno al interés por la sociología. No podemos, sin embargo, afirmar que estas revistas se leyeran y, en su caso, se asimilaran; nadie puede hacerlo. Simplemente puede presentarse el hecho constatable de que ello fue posible; de que en el Instituto de Reformas Sociales, entre los años 1906 y 1912 (seguramente desde el mismo 1903 y hasta el 1924, aunque ello no lo documente el trabajo de Posada), quien por allí anduviera tuvo físicamente al alcance de la mano obras de Weber, de Simmel, de Ward, de Small, etcétera. El análisis de la producción de nuestros primeros sociólogos, en cualquier caso, atestigua - por medio de la bibliografía que en sus obras incorporaban sobre todo- que en muchos de ellos pesaron estas lecturas, y un ejemplo claro lo ofrece la obra de Posada. Así lo argumenta, por ejemplo, Luis Saavedra (1991) en El pensamiento sociológico español, donde atribuye a las lecturas de Simmel _ ¿por qué no las recibidas en el Instituto? - el que Posada «se decanta por una sociología experimental, aunque con ciertos matices que aminoran la significación de los postulados más positivistas. Y esas puntualizaciones [continúa Saavedra] son las que nos recuerdan las lecturas benéficas de un Simmel [...] cuando habla de la sociología como una ciencia "especial", profundamente afectada por la sustancia de sus componentes» (Saavedra, 1991: 140).

Queda claro que la biblioteca del Instituto recibía regularmente un buen 
número de publicaciones periódicas internacionales en el campo de la sociología. A ellas sumemos ahora otro aspecto de su biblioteca: los fondos bibliográficos en materia de libros. Aunque resulta difícil seguir el rastro de los libros de los que el Instituto disponía al disolverse en 1924 — de los que Palacio Morena, en su libro La institucionalización de la reforma social en España (18831924), afirma ser unos 50.000 volúmenes (Palacio Morena, 1988: 314)—, contamos en esta ocasión con una herramienta valiosísima. Se trata del libro de Julio Sempere Olivares (1956) Información bibliográfica. Obras de carácter social existentes en la "Sección de Estudios y Biblioteca» del Ministerio de Trabajo, obra que, prologada por León Martín Granizo, es en realidad un exhaustivo catálogo de los fondos en la materia de dicha biblioteca ${ }^{17}$.

Afirmamos que resulta difícil aun así seguir la pista de aquellas obras que fueron de consulta para los miembros del Instituto - pero también para el público en general ya que se trataba de una biblioteca abierta-, porque de los más de setecientos títulos que en el catálogo mencionado aparecen bajo la materia "sociología», algunos de ellos están editados con posterioridad a 1924, circunstancia que imposibilita el que hayan sido adquiridos por la biblioteca del Instituto. Existen, sin embargo, muchos otros que, con toda probabilidad, sí pertenecieron a la misma, ya que sus temáticas y fechas de edición (muchas de ellas primeras ediciones) coinciden con el período de vigencia de la institución. En cualquier caso, todas las obras de las que a continuación vamos a ofrecer una muestra son ejemplares (en español, inglés, francés, alemán e italiano) que en el año 1956 estaban en España - y aún lo están—, en la biblioteca del Ministerio de Trabajo, a disposición de los estudiosos de las ciencias sociales.

Insistimos en la dificultad de seguir la pista a los libros cuya adquisición se debió al Instituto de Reformas Sociales de entre todos aquellos que figuran en el catálogo de Sempere. No nos parece, sin embargo, que sea muy aventurado el suponer que los que se editaron antes de 1924 (aproximadamente la mitad, unos 350, que si sumamos los aparecidos sin fecha —omisión más frecuente en obras antiguas - alcanzan casi las cuatro centenas) pudieron pertenecer a aquél. De entre ellos, casi cien están en inglés, más de ochenta en francés, unos setenta en castellano, sesenta en alemán y casi cuarenta en italiano; todos ellos publicados con anterioridad a 1924 y que figuran en el catálogo de Sempere dentro del epígrafe «sociología», lo que quiere decir que si sondeáramos en profundidad dentro de otras temáticas, como "política social», "urbanismo» o «migración», el número de obras cuyos contenidos puedan clasificarse de «sociológicos» seguramente se multiplicaría.

Pero más allá de la descripción numérica, que por sí misma da cuenta de una buena cantidad de información sociológica al alcance de los estudiosos españoles, señalemos algunos de los autores y obras que, por estar contenidas en la biblioteca que fuera la del Instituto, nos instruyen sobre el grado de

${ }^{17}$ El Instituto de Reformas Sociales, al extinguirse como tal en 1924, se incorporó al Ministerio de Trabajo, lugar al que pasaron sus fondos documentales (cf. Palacio Morena, 1988). 
información de los sociólogos españoles de esas primeras generaciones o, al menos y desde luego, de la posibilidad de tal información. Así, entre los libros en castellano, además de los distintos trabajos de Giner de los Ríos, Sales y Ferré, Azcárate o Posada, encontramos también las obras firmadas por el propio Instituto, así como los discursos y contestaciones de la Real Academia de Ciencias Morales y Políticas y distintas aportaciones de sociólogos iberoamericanos que escribieron a principios de siglo, como puedan ser las de Raúl Orgaz o Ernesto Quesada. Pero destaca también la cantidad y variedad de obras extranjeras traducidas al castellano con anterioridad al año 1924. Algunas de ellas son responsabilidad directa de Adolfo Posada, pero sin duda también respuesta al interés que en la sociología mostraron estos primeros estudiosos españoles. Así, los varios trabajos de Lester Ward, de Gabriel Tarde, de Herbert Spencer (de quien en esos primeros años del siglo aparecen traducidas seis obras en la editorial La España Moderna), de Greef o de Giddings, muchos de ellos en editoriales que fueron pioneras y que se recordarán, como las de Fernando Fe o Daniel Jorro en Madrid, Henrich y Cía. en Barcelona o Sempere en Valencia.

Entre estas obras a las que nos referimos, vemos aparecer en francés contribuciones a la sociología como De la division du Travail Social o Le Suicide, de Emile Durkheim, ambas en sus ediciones originales (de 1893 y 1897, respectivamente); los seis volúmenes de las Ouvres Complètes de Le Play (en su edición de 1878-79); la Psychologie des temps nouveaux de Le Bon; La mentalité primiti$v e$, de Levy-Bruhl, o distintos trabajos de Gabriel Tarde o, en otro contexto, de Adolphe Quetelet, por mencionar sólo algunas de ellas. En cuanto a las obras anglosajonas, la lista es tan larga (roza el centenar) que sólo una muestra no da cuenta de lo que la biblioteca tenía en sus fondos. Aun así, destaquemos por su importancia trabajos de Charles H. Cooley como los seminales Human Nature and the Social Order o Social Organization (en sus ediciones respectivas de 1902 y 1909); la no menos significativa Introduction to the Science of Sociology, de Park y Burgess, de 1921; el manual de 1908 Social Psichology, de Ross, considerado por muchos analistas como el primer manual de psicología social de inspiración sociológica; un trabajo de William I. Thomas de 1907; o la edición de 1905 de la célebre The Theory of Leisure Class, de Thorstein Veblen, que no sería traducida al castellano hasta cuarenta años después en México. En cuanto a las obras en alemán e italiano, aunque representadas en menor número, Weber, Simmel, Tönnies, Schaffle o Von Wiese quedan también representados; otro tanto ocurre con los italianos Michels, Ferrari o Squillace.

\section{c) Las investigaciones del IRS}

Creo que ha quedado suficientemente mostrado que el IRS contaba, para la época, con las fuentes fundamentales para el estudio sociológico, pudiendo sin exagerar calificar de excepcional su recepción de obras extranjeras, hecho que, aun con los lógicos retrasos en las comunicaciones y transportes de la época, le 
capacitó para estar a la altura del saber mundial de su tiempo. En el ya citado trabajo de Palacio Morena (1988: 322) puede leerse que «una de las primeras tareas que emprende el Instituto de Reformas Sociales es la de establecer relaciones con las Oficinas y Departamentos extranjeros de carácter análogo al español. El objetivo más inmediato es el intercambio de publicaciones e informes de interés mutuo». A tal efecto, al poco de crearse el Instituto se redactó una circular que, como en el mismo lugar se señala, fue remitida a entidades similares o análogas a la española de Francia, Inglaterra, Italia, Bélgica, Austria, Rusia, Suecia, Dinamarca o Estados Unidos (cf. ibidem). La proyección internacional se constata asimismo en la asistencia a congresos y reuniones internacionales, de cuyos contenidos se informaba posteriormente y era usual dar noticia en el Boletín, donde queda constancia de la presencia de representantes españoles. De entre ellos destaca una vez más el activo Posada, quien en 1910 viaja por distintos países de América donde «imparte diversas conferencias y cursos en Argentina, Paraguay y Chile» (Palacio Morena, 1988: 324), además de asistir a la primera Conferencia de Trabajo, celebrada en Washington en 1919. Añadamos que, como señala el mismo autor (op. cit.: 325), el Instituto mantenía «relaciones con el Instituto Internacional de Bibliografía de Bruselas, la Universidad de California o el British Institute of Social Service».

Sin embargo y aun siendo excepcional el dominio de tantas y tan precisas fuentes de saber sociológico, podría quizá concluirse que el IRS simplemente contó con una biblioteca institucional con una muy documentada sección de sociología, quedando su interés limitado a la historia de la biblioteconomía española. Pero, como enseguida veremos, esta inmensa documentación sociológica tuvo un correlato en el interés investigador, o éste en aquélla, que tanto da, pues lo que importa resaltar es que ambos aspectos del saber científico social quedaron más que cubiertos en el IRS.

Donde, a mi entender, se revela toda la proyección investigadora de los hombres del Instituto es en los así llamados por el Instituto «estudios monográficos». En aquellos que así describe Juan Ignacio Palacio Morena: «El Instituto elabora informes a raíz de ciertos sucesos (catástrofes mineras, huelgas que afectan directamente al orden público, etc.) en los que, por su especial importancia y complejidad, se estima conveniente un estudio pormenorizado de sus causas y una reflexión sobre las medidas que se deben instrumentar para corregirlas» (Palacio Morena, 1988: 259). En esas circunstancias se comisionaba uno o más inspectores para que, en el lugar de los hechos objeto de interés, investigaran cuanto pudiera ser necesario saber para conocer el problema. La motivación reformista del Instituto es aquí donde mejor se ejemplifica. Conocer para solucionar. De entre estos informes monográficos, que, además de aparecer noticiados en el Boletín de la Institución, solían generar una publicación en forma de memoria, voy a tomar tres ejemplos en los que nos detendremos. En ellos, así me lo parece, se encuentran interesantes elementos metodológicos que les hacen acreedores de un puesto de relevancia en la historia de la investigación sociológica española. 
Comencemos por un trabajo de José Marvá, jefe de la sección segunda del Instituto, quien en 1904, según él mismo explica, fue "comisionado por el Instituto de Reformas Sociales para informar acerca de las causas de la catástrofe ocurrida en las hulleras de Villanueva de las Minas el día 28 de Abril del año actual» (Marvá, 1904: 1). Se trataba de un gravísimo accidente en unas minas sevillanas donde, al proceder a perforar un túnel nuevo de trayectoria ascendente, ocurrió una explosión en la que murieron varios mineros. Entre ellos, dos jóvenes de dieciséis años. Es éste un claro caso de estudio para el Instituto, en el que se ven involucrados aspectos como el cumplimiento de las medidas de seguridad en las minas (funcionamiento de aparatos ventiladores, prohibición de fumar), la contratación de jóvenes (prohibida para algunas tareas de alta peligrosidad), la responsabilidad de los capataces en el accidente, etc.

Para la investigación y posterior dictamen de las causas de la catástrofe se envió, como decimos, a José Marvá, quien expone «el resultado de mis investigaciones, en las que he utilizado las fuentes de información siguientes ...» (Marvá, 1904: 1). Antes de presentar las fuentes que Marvá explicita, hagamos notar que el hecho de hacerlo ya indica de por sí cierto talante de comunicación de procedimientos que es de valorar. Pero continuemos leyendo. En primer lugar, dice haber utilizado «informaciones obtenidas directamente, interrogando a mineros, ex mineros y ex capataces que abandonaron el trabajo de las minas para dedicarse al pequeño comercio en Villanueva» (ibidem). Es decir, conversaciones directas (entrevistas) con quienes están directamente implicados en el objeto de estudio: los mineros; y también con quienes, conociendo la labor minera, al haberla abandonado, pueden ofrecer una información (desde cierta distancia) más equilibrada, por no pertenecer ya al colectivo perjudicado.

Pero continuemos, porque Marvá añade a sus fuentes «datos suministrados por el Ingeniero director de las minas Sr. Tombelaine, por el Médico de las minas D. Guillermo Fernández, e informes que obtuve en conferencias con Ingenieros subalternos franceses y con capataces» (ibidem). Es decir, sometimiento a "juicio de experto», diríamos hoy, o, desde luego y de manera clarísima, consulta a las personas ilustres de la localidad (con palmaria resonancia al «método de las personas ilustres» de Le Play). En una localidad (una mina), el alcalde, diríamos, es el ingeniero jefe.

Pero no acaba ahí la labor investigadora de Marvá, pues éste añade a sus fuentes el «examen directo, practicado por mí, de los lugares, minas y edificios. A este efecto, bajé a las minas, [...]; las dependencias del economato; los alojamientos del obrero, ya las casas que construye para ellos la empresa, ya las chozas y cuevas; la enfermería; [...] la farmacia; escuelas de niños, etc. [y concluye] Del resultado de mis investigaciones doy cuenta en las páginas que siguen" (ibidem). Así, pues, además de las conversaciones con los mineros, de los informes recibidos de boca de expertos y "personas ilustres», Marvá considera necesario hacer trabajo de observación, trabajo de campo. El accidente fue en un túnel incorrectamente perforado (parece ser que había de ser en trayectoria descendente para haber evitado la concentración de partículas altamente infla- 
mables de carbón de hulla en el aire), pero Marvá / investigador de campo considera necesario visitar "las chozas y cuevas» donde viven los mineros, "el economato» donde se suministran de lo necesario para vivir, «la enfermería» donde se les atiende de las dolencias, el colegio de sus hijos... Parece evidente que, en el ánimo de Marvá, en su concepción de lo que era necesario conocer para solucionar el problema, gravitaba algo más que el informe técnico de la explosión ocurrida.

Que no se trata el informe de Marvá del producto de una personalidad aislada o excepcional, sino más bien de una práctica habitual de los hombres del Instituto, queda apuntado con la siguiente referencia. A causa de una problemática diferente (una huelga), pero en el mismo contexto (unas minas), se encargó ese mismo año una comisión para el estudio de las minas de Vizcaya. Estas comisiones de las que estamos presentando algunos ejemplos trabajaban con celeridad pues, como hemos ya señalado, se trataba de expediciones a lugares de conflicto donde urge un dictamen para emprender las medidas pertinentes. Ésa era la labor del Instituto.

La necesidad de afrontar con prontitud un problema candente se destaca en el informe al que aludimos a continuación: «El Instituto, con fecha 9 [de noviembre de 1904], nombró la Comisión indicada, compuesta por los Vocales D. Rafael Salillas y D. Eduardo Sanz y Escartín, y D. Julio Puyol, como secretario de la misma, quienes, al día siguiente, sin pérdida de tiempo, salieron de Madrid con dirección a Bilbao a cumplir el encargo que se les había confiado» (Salillas, Sanz y Escartín, y Puyol, 1904: 5-6). Esa premura, como apuntamos, se debía a la necesidad de estudiar y conocer, para informar de ello, un conflicto minero en Vizcaya. Las causas de aquél fueron la reivindicación de cantinas obligatorias y el pago semanal de los jornales.

En el informe presentado por los enviados del Instituto se encuentran datos de interés que contribuyen a perfilar la metodología o manera de afrontar las investigaciones encargadas que ya hemos presentado en pluma de Marvá. Allí leemos, en el apartado "Trabajos realizados por la Comisión», que: «La Comisión del Instituto creyó desde el primer instante que el mejor procedimiento que podía seguir era el de la información directa; es decir, las conferencias con los obreros en los mismos lugares en que prestan el trabajo; la inspección de sus alojamientos y de las tiendas en que se proveen de lo necesario para la vida; las visitas a las fábricas, hospitales y asilos, etc. etc., y así, tan pronto como llegó a Bilbao, púsose de acuerdo con las Autoridades, con los obreros, patronos, contratistas de minas y Compañías de ferrocarriles, con objeto de comenzar cuanto antes sus trabajos» (op. cit.: 6). Las coincidencias entre el procedimiento de la comisión formada por estas tres personas del Instituto y el de Marvá ya presentado disipan cualquier duda acerca de la posible excepcionalidad en la metodología de Marvá que anteriormente presentamos. Ésa era, según nos parece, la manera de investigar del Instituto: información directa, observación del terreno, recurso a las autoridades competentes, etc. 
Pero, además de la información directa, que los autores enumeran detalladamente ("visita a las minas de la zona», "visita al Asilo de San Fernando», "visita a las fábricas de Altos Hornos», "conferencia con los representantes del Círculo Minero", etc.; cf. en la obra citada, 6 y 7), se presenta un apartado de "Información documental» que presenta interés y cierta novedad con respecto al trabajo de Marvá. Así es; nada más llegar a Bilbao, afirman estos autores que se "dirigió una circular a los patronos de las minas para que contestasen a las siete preguntas que en el cuestionario se formulaban. Pasan de setenta las respuestas recibidas, que serán citadas y tenidas en cuenta en los lugares oportunos de este informe» (ibidem). Nos encontramos, pues, con un primer contacto con la realidad objeto de estudio, el suministro de un cuestionario (bien que breve) para analizar sus resultados. Ya señalamos que Azcárate había realizado en la Comisión de Reformas Sociales —antecesora del Instituto, en el que Azcárate, dicho sea de paso, continuó su labor hasta su propia muerte en 1917 - una tarea semejante. Estos datos complementan a la observación directa y entrevistas personales, o éstas a aquéllas, que tanto da. ¿Proto-triangulación de técnicas? Quizá sería exagerado responder afirmativamente a esa pregunta. Sin embargo, contamos con otro elemento que se presenta como trabajo realizado por la comisión.

Así es, los autores del informe añaden: «Además de esto, la Comisión del Instituto ha procurado recoger otros muchos documentos, tales como tablas de salarios y de horas de trabajo, aumento que han tenido los jornales y los artículos de primera necesidad en los últimos diez años, reglamento de explotación, número y clase de asociaciones obreras [...] en una palabra, todas aquellas noticias que puedan contribuir al mayor esclarecimiento de la cuestión y servir de comprobante a las afirmaciones que se hagan en el presente trabajo.» (op. cit.: 7 y 8). Así, pues, la observación directa sobre el terreno, las entrevistas a los protagonistas, la implementación de un cuestionario y el análisis de datos secundarios nos parecen elementos suficientes para atribuir a estas investigaciones carácter sociológico.

Pero cabe cuestionarse esta última afirmación y preguntarse si es posible sostener, después de estas notas sobre las fuentes que Marvá expresa haber utilizado y sobre las otras de Sanz y Escartín, Salillas y Puyol, que estos hombres del Instituto tenían espíritu sociológico, además del claro talante reformador. ¿Fue más allá su tarea de la de meros inspectores de trabajo, o expertos de un órgano de información al Gobierno? Parece que así fue, y con la siguiente referencia creo poder demostrarlo.

Ese mismo año 1904, el Instituto de Reformas Sociales, percatado de que «en una importantísima comarca agrícola española advertíase casi por primera vez agitación entre la gente obrera del campo, siempre pacífica hasta ahora, o por ignorante, o por resignada, o por las dos cosas a un tiempo" (Álvarez Buylla, 1904: 5), envió una comisión para su estudio. En este caso se encomendó la tarea a la sección tercera (la que se ocupaba de la información estadística) y a tal efecto fue comisionado el jefe de dicha sección, Adolfo Álvarez Buylla. 
Aunque vamos a subrayar los aspectos metodológicos de la investigación de Buylla, no parece inoportuno señalar que su trabajo sobre el problema agrario castellano tiene también en cuanto a contenidos una relevancia singular. Sobre la memoria de la información agraria en ambas Castillas existe una monografía que así lo demuestra. Publicada en 1977 con el título Miseria y conciencia del campesino castellano (Instituto de Reformas Sociales, 1977) y analizada por Julio Arostegui, contiene esta obra el trabajo de Álvarez Buylla, además de un amplio estudio preliminar y un análisis textual de varios fragmentos que muestran el interés histórico del texto.

El que fuera catedrático de Economía en la Facultad de Derecho de Oviedo, Adolfo Álvarez Buylla y González Alegre, que años después (1917) sería recibido como académico en la Real Academia de Ciencias Morales y Políticas con un discurso que versó sobre, precisamente, la reforma social en España, presenta así, en esta memoria de 1904, el conjunto del trabajo. En las palabras que a continuación se reproducen permea claramente su concepción sobre la reforma social y a qué organismo corresponde suministrar los datos para realizarla. Así, leemos: «En pocas ocasiones en España han correspondido tan pronto las iniciativas del Gobierno a las manifestaciones de la opinión, y por esta vez bien claro se ve que el Estado pretende, empleando el procedimiento más adecuado para que el remedio legislativo tenga verdadera eficacia, conocer con todo detalle el mal y sus causas, y conocerlo por boca de quien lo siente, a quien también consulta sobre la medicina apropiada, valiéndose del órgano especialmente creado para llevar a cabo estas funciones, el Instituto de Reformas Sociales; que esto, y no otra cosa, significa la información decretada" (Álvarez Buylla, 1904: 6 y 7). Más allá del organicismo terminológico que desprende el vocabulario utilizado por Álvarez Buylla, hagamos notar que, además de indicar la competencia del Instituto de Reformas Sociales, el acento queda puesto sobre "conocerlo por boca de quien lo siente», pues ahí radica el método que Álvarez Buylla anuncia como más apropiado.

Pero hay que repetir la pregunta anterior: ¡es esto sociología?, o mejor: ¿se sentían sociólogos estos investigadores? Álvarez Buylla lo contesta sin dejar el menor resquicio de duda en el párrafo siguiente, el que lleva por subtítulo «Carácter de la Información» (Álvarez Buylla, 1904: 7). Allí se puede leer: «No es el caso teorizar acerca del carácter y de la importancia de este procedimiento sociológico, que es estadística, historia y monografía a un tiempo; puesto que anima, vivifica el dato numérico con la expresión de cuanto, en la existencia de la persona individual y de la persona social, escapa a la rigidez de aquél, y que constituye su interesante trama; los sentimientos (deseos, aspiraciones, esperanzas, necesidades, pasiones, entusiasmos), los pensamientos (ideas, razonamientos, dudas, errores), las voliciones (impulsos, motivos, hábitos, virtudes, vicios)» (op. cit.: 7; énfasis nuestro). Ya lo hemos subrayado en el texto transcrito: Álvarez Buylla no quiere teorizar sobre su procedimiento, pero éste es "genuinamente sociológico». Una combinación de técnicas que "anima[n], vivifica[n] el dato numérico», y consiguen huir de «la rigidez de aquél». 
Si el trabajo señalado de Marvá superaba en sus intereses el informe técnico de un accidente minero, y otro tanto puede afirmarse del informe de Salillas, Sanz y Escartín, y Puyol, esta presentación de Álvarez Buylla creo que representa igualmente algo más que la introducción al estudio jurídico/institucional de un conflicto huelguista. No termina aquí, sin embargo, el interés del trabajo del que fue académico de Ciencias Morales y Políticas, pues a la información relatada tras la presentación que se ha transcrito añade una serie de apéndices de documentación también consultada por él para su estudio. Destaquemos que, entre otros documentos (telegramas oficiales al Instituto, propuestas de organizaciones obreras, etc.; cf. en Álvarez Buylla, 1904: 66 y ss.) -que ya hemos visto utilizar a Salillas, Sanz y Escartín, y Puyol_-, aparecen transcritos en la memoria de Álvarez Buylla lo que denomina «Noticias y juicios de los periódicos de la provincia acerca del movimiento obrero agrícola» (op. cit.: 67). Es decir, estaríamos ante una temprana utilización de este tipo de datos secundarios extraídos de la prensa, datos textuales, no numéricos, por un sociólogo que contempla los comentarios de los periódicos regionales (lo hace en todas las provincias a las que se refiere su estudio) como valiosa información. Aun sin estudiar en detalle cómo incorpora esos datos a su informe, puede afirmarse, sin embargo, que la mera presencia de los mismos en un apéndice junto a otros documentos de interés recuerda, sin un esfuerzo imaginativo exagerado, al actual análisis de contenido de los medios de comunicación escritos. Más que si se llegó a realizar o no, importa —así me lo parecela intención que subyace al hecho de tenerlos en cuenta como datos de su informe.

\section{CONCLUSIONES}

Es muy tentador catalogar en un mismo saco todo lo anterior al momento de la institucionalización más o menos formal de la sociología en este o en cualquier país como pre-sociología, preámbulos, pioneros, precursores, precedentes o cualquier otro calificativo que la despache como de mero interés arqueológico, pintoresco, etc., dispensándose a uno mismo, entre otras cosas, de la tarea de su estudio. Ya lo señaló J. F. Marsal a propósito de la sociología en Argentina y en otros países iberoamericanos donde la estudió: "Creo que a estas alturas no se puede poner la larga etapa de conocimiento social latinoamericano, que va desde los primeros balbuceos en la Argentina colonial y la Independencia hasta mediados del siglo XX, en un solo paquete al que se le llame "pre-Sociología" (...) Porque aunque sea con mejor ropaje académico, esta denominación no hace más que repetir la equivocada desviación "progresista" de las historias de la sociología latinoamericana (...) En todas ellas, incluida la reciente de Solari, la primera etapa, la etapa precientífica o presociológica, viene a ser una categoría residual e indiscriminada» (Marsal, 1979b: 21) 
También es cierto, y en palabras del mismo autor pero distinta obra, que «no se puede exagerar lo que en España había» (Marsal y Oltra, 1980: 29). Pero entre el tratamiento indiscriminado como presociológico y la exacerbación desmesurada hay numerosas tonalidades intermedias. Según me parece, no es exagerado aquí afirmar que la ignorancia del Instituto de Reformas Sociales, de sus hombres, de sus obras e investigaciones, deja huérfana la historia de nuestra sociología. Sin su conocimiento y estudio no podrá siquiera analizarse su posible papel y trascendencia.

El debate sobre cuál sea la fecha a partir de la cual es razonable hablar de sociología española sigue abierto y, probablemente, nunca se cerrará. Aunque hoy no mantiene la actualidad y polémica que en otro tiempo alcanzó, lo cierto parece ser que tras las distintas posturas posibles (que oscilan en más de medio siglo de distancia) se encuentran concepciones también muy distintas de la propia sociología, lo que dificulta un posible acuerdo o consenso. El debate no es, pues, de interpretación histórica, sino más bien entre posiciones teórico-epistemológicas, lo que, además de complicarlo inmensamente, también lo convierte en mucho más interesante. Lo que se ventila, y creo que en esto habrá acuerdo, no es un baile de fechas; los años 1875, 1899, 1903, 1914, 1953 ó 1958 han sido todos ellos propuestos como del comienzo de la sociología española. Su interés es meramente anecdótico si lo enfrentamos a las distintas concepciones teóricas que tras esas elecciones se enmascaran o subyacen. Pero para poner en juego esas concepciones es necesario primero conocer y estudiar los elementos presentes en tal o cual momento que se esté analizando. Los datos ofrecidos acerca del Instituto de Reformas Sociales creo que por sí mismos bastan para sugerir un análisis más profundo de su posible papel en la historia de nuestra sociología ${ }^{18}$. Retomando el argumento de la presentación, si el Instituto de Reformas Sociales es considerado un dato de historia cuatro, es hora de incorporarlo a una nueva historia uno que, de paso, contribuya a poner en su lugar a tantas historias ocho que nos confunden y enredan. El centenario que durante 1999 se está conmemorando en la sociología española no parece mala excusa para que así sea.

${ }_{18}$ Quisiera señalar que no es el IRS el único astro dado por muerto en el firmamento de la historia de la sociología española y que, sin embargo, aún calienta e ilumina, ya que han sido otros varios los que a lo largo de la investigación que he realizado me han sido descubietos y quizá oportunidades futuras me permitan ir desgranándolos a la altura del tratamiento que, en mi opinión, merecen. 


\section{BIBLIOGRAFÍA}

Álvarez Buylla, A. (1904): Memoria acerca de la información agraria en ambas Castillas, Madrid, Instituto de Reformas Sociales.

Álvarez-Uría, F. (ed.) (1997): Jesús Ibánez, teoría y práctica, Madrid, Endymion.

Ayala, F. (1984) [1982]: Recuerdos y olvidos (2 vols.), Madrid, Alianza.

AzCÁrate, P. de (1969): Gumersindo de Azcárate, Madrid, Tecnos.

BARnes, H. E., y BECKer, H. (1984) [1938]: Historia del pensamiento social. Corrientes sociológicas en los distintos países (Tomo 2), México, Fondo de Cultura Económica.

Baudrillard, J. (1984) [1978]: Cultura y Simulacro, Barcelona, Kairós.

Borx Selva, E. (1961): «Sociología», en Enciclopedia Universal Espasa, Suplemento 1957-58, Madrid, Espasa-Calpe.

Calle, M. D. de la (1989): La Comisión de Reformas Sociales (1883-1903), Madrid, Ministerio de Trabajo y Seguridad Social.

CAMPO, S. del (1997): "Cánovas del Castillo y el nacimiento de la Sociología española», en VV.AA., Antonio Cánovas del Castillo. Homenaje y memoria de la Real Academia de Ciencias Morales y Politicas (1897-1997), Madrid, Real Academia de Ciencias Morales y Políticas, pp. 319-331.

CANCiO, M. (1995): «La institucionalización de la Sociología: El caso del CIS, de la ideología proclamada a la práctica real», Politica y Sociedad, 17, pp. 125-144.

Carrasco i Calvo, S. (1980): «Trenta anys d'activitat docent i d'investigació social. L'Institut Catòlic d'Estudis Socials de Barcelona», Perspectiva Social, 16, pp. 117-140.

CASTillo, J. (1973): «Apuntes para una historia de la sociología española», en G. DunCan MitCHELl, Historia de la sociología. Tomo II (Apéndice), Madrid, Ediciones Guadarrama, pp. 109-144.

Cazorla, J. (1967): «Estudios empíricos de sociología española», Anales de Sociología, 3, pp. 146-156.

COMISIÓN DE REFORMAS SOCIALES (1985): Reformas Sociales: información oral y escrita publicada de 1889 a 1893 (edición al cuidado e introducción de Santiago Castillo), Madrid, Centro de Publicaciones del Ministerio de Trabajo y Seguridad Social (la edición original fue en Madrid, Imprenta de Manuel Minuesa de los Ríos, 1889-1893).

DíAZ, E. (1979): «Reformismo social krausista: Gumersindo de Azcárate», en C. Lida e I. Zavala (eds.), La revolución de 1868, Nueva York, pp. 239-253.

- (1989) [1972]: La filosofía social del krausismo español, Madrid, Debate.

- (1996): «Joaquín Costa: ¿Regeneración sin parlamento?», Boletín de la Institución Libre de Enseñanza, 2. ${ }^{\text {a }}$ época, 24-25, pp. 133-142.

Dí́zZ NiCOlÁs, J., et al. (1989) [1984]: Cincuenta años de sociología en España, Málaga, Universidad de Málaga.

GINER, S. (1984): «Sobre mi trabajo», Anthropos, 36, pp. 4-12.

Giner, S., y Moreno, L. (comps.) (1990): Sociología en España, Madrid, Centro de Investigaciones Sociológicas.

Gómez Arboleya, E. (1958): «Sociología en España», Revista de Estudios Políticos, 98, pp. 4783.

Hermet, G. (1968-69): «La sociología empírica en España», Anales de Sociología, 4-5, pp. 268301.

IBÁÑEZ, J. (1990): «Autopercepción intelectual de un proceso histórico», Anthropos, 113, pp. 9-25.

IbÁÑEz, J. (dir.), y Reyes, R. (ed.) (1992): Las Ciencias sociales en España. Vol. 1: Sociología, Madrid, Universidad Complutense.

Instituto De Reformas Sociales (1919): El Instituto de Reformas Sociales, Madrid, Sobrinos de la Sucesora de M. Minuesa de los Ríos.

- (1977): Miseria y conciencia del campesino castellano, Madrid, Narcea.

JEREZ Mir, R. (1981): «Significación social del pensamiento y la obra de Manuel Sales y Ferré (1843-1910)», Revista Española de Investigaciones Sociológicas, 39, pp. 329-352. 
Jiménez Blanco, J. (1984): «Enrique Gómez Arboleya (1910-1959)», Cuadernos de Ciencia Política y Sociología, 15-16, pp. 37-41.

- (1985): «Diez años de sociología en España (1974-1984)», Cuenta y Razón, 19, pp. 133-142.

lamo de Espinosa, E.; González García, J. M., y Torres Albero, C. (1994): La sociología del conocimiento y de la ciencia, Madrid, Alianza.

Laporta, F. J. (1974): Adolfo Posada: Politica y sociología en la crisis del liberalismo español, Madrid, Cuadernos para el Diálogo.

LinZ, J. J. (1994): La sociología. Hablando con Juan Linz, Madrid, Acento Editorial.

- (1997): «Between nations and disciplines: personal experience and intellectual understanding of societies and political regims", en Hans Daalder (ed.), Comparative European Politics: The Story of a Profession, Londres y Washington, Pinter, pp. 101-114.

López Coira, M. M. (1986): El pensamiento social de Severino Aznar (1870-1959), Madrid, Universidad Complutense.

Maestre Vives, T. (1962): «Sociedad y nación en Sales y Ferré», Revista de Estudios Políticos, 124, pp. 133-146.

Marín, X., et al. (1993): «El discurs cientific y els processos d'institucionalió científica a la sociologia. El cas de la revista Papers (1973-1993)», Papers, 42, pp. 49-104.

MARSAL, J. F. (1979a): Pensar bajo el franquismo, Barcelona, Península.

- (1979b): Dependencia e independencia, Madrid, CIS.

Marsal, J. F., y Oltra, B. (eds.) (1980): Nuestra Sociedad. Introducción a la Sociología, Barcelona, Vicens Vives.

Martín-Granizo, L. (1947): El Instituto de Reformas Sociales y sus hombres, Madrid, Patronato de la Escuela Social de Madrid.

Martín LÓpez, E. (1992): "La concepción de la Sociología en Sales y Ferré», en C. Moya y otros (comps.), Escritos de Teoría Sociológica en Homenaje a Luis Rodríguez Zúñiga, Madrid, Centro de Investigaciones Sociológicas, pp. 649-669.

MARVÁ, J. (1904): Memoria acerca de la visita de inspección girada a las minas de Villanueva (Sevilla), Madrid, Instituto de Reformas Sociales.

MendizÁbal, A. (1965) [1956]: "La sociología en España», en G. Gurvitch y W. E. Moore (eds.), Sociología del siglo XX, tomo II, Madrid, Ateneo, pp. 343-357.

Miguel, A. de (1972): Sociología o subversión, Barcelona, Plaza y Janés.

- (1987): "Las primeras encuestas en España», en VV.AA., Política y Sociedad. Estudios en homenaje a Francisco Murillo Ferrol, Madrid, Centro de Investigaciones Sociológicas y Centro de Estudios Constitucionales, vol. II, pp. 703-709.

Miguel, J. M. de, y Moyer, M. G. (1979): «Sociology in Spain», Current Sociology, 27 (1), monográfico.

NúNEEZ, D. (1973): «La sociología en España (1875-1914)», Revista Internacional de Sociología, 5-6, pp. 139-175.

- (1987) [1975]: La mentalidad positiva en España, Madrid, Universidad Autónoma.

NúNEz ENCABO, M. (1976): Manuel Sales y Ferré: los origenes de la sociología en España, Madrid, Cuadernos para el Diálogo.

ORTí, A. (1996): «Para la regeneración de la memoria democrática: Conmemoración en el Instituto Francés de los 30 años y un día... de la Escuela Crítica de Ciencias Sociales de CEISA, un lugar de encuentro de la vanguardia cultural antifranquista de los años 1960" (multicopiado).

Palacio Morena, J. I. (1988): La institucionalización de la reforma social en España (18831924), Madrid, Ministerio de Trabajo y Seguridad Social.

Perpiñá Rodríguez, A. (1968): «La sociología en España», Revista Iberoamericana de Seguridad Social, 6, pp. 1171-1189.

Perspectiva Social (1979): «El Laboratori de Sociologia de l'ICESB», Perspectiva Social, 13, pp. 131-141.

PInILla de las Heras, E. (1996): La memoria inquieta: Autobiografía sociológica de los años difíciles 1935-1959, Madrid, Centro de Investigaciones Sociológicas. 
POSADA, A. (1990): «La sociología en España», REIS, 52, pp. 161-194 (original de 1899).

Ríos, F. de los (1949) [1930]: «Spain and Portugal», en Encyclopaedia of the Social Sciences, tomo I, Nueva York, The Macmillan Company, pp. 295-300.

Rodríguez IbÁNEZ, J. E. (1996): «Desde un fin-de-siècle a otro: Un obligado recuerdo a Manuel Sales y Ferré», REIS, 76, pp. 221-225.

Rodríguez ZúNiga, L., y SAlcedo, J. (1991): "Veinte años de sociología», Sistema, 100, pp. 103-107.

SAAVEDRA, L. (1991): El pensamiento sociológico español, Madrid, Taurus.

SÁnchez Agesta, L. (1983): «Las memorias de Adolfo Posada. Anécdotas y recuerdos en la España de la Restauración», Cuenta y Razón, 13, pp. 25-32.

SANZ y Escartín, E.; Salillas, R., y PuYOl y Alonso, J. (1904): Informe referente a las minas de Vizcaya, Madrid, Instituto de Reformas Sociales.

Sarabia, B., y Zarco, J. (1997): Metodología cualitativa en España, Madrid, Centro de Investigaciones Sociológicas.

SARries SANZ, L. (1986): "Dimensión sociológica del pensamiento de Joaquín Costa», Annales, 3, pp. 221-225.

SAstre, V. J. (1976): Las ciencias sociales en España, Madrid, Seminario de Investigaciones Sociológicas (monográfico de la revista Documentación Social, n. ${ }^{\circ} 24$ ).

Seminario de TeOría Sociológica (1981): «Bibliografía en español de los clásicos de la sociología», Papers, 15, pp. 153-199.

Sempere Olivares, J. (1956): Información bibliográfica. Obras de carácter social existentes en la "Sección de Estudios y Biblioteca» del Ministerio de Trabajo, Madrid, Servicio de Estudios y Formación Social.

Torregrosa, J. R. (1964): «El pensamiento político de D. Gumersindo de Azcárate», Revista de Estudios Políticos, 135-136, pp.

Vidal Beneyto, J. (1973): «Sociología y garbanzos», en A. DE Miguel, Homo sociologicus hispanicus, Barcelona, Barral Editores (Epílogo).

VV.AA. (1971): Sociología española de los años setenta, Madrid, Confederación Española de Cajas de Ahorros.

- (1974): "Conversaciones sobre la situación de la Sociología en la Universidad española. I Simposio en Bellaterra", Papers, pp. 179-200.

- (1988): Homenaje a Enrique Gómez Arboleya, 1910-1959, Granada, Ayuntamiento de Granada y Departamento de Sociología y Psicología Social de la Universidad de Granada.

\section{ABSTRACT}

Using three definitions of the word "history», this paper presents some of the key works on the history of Spanish sociology, some of the events marking its trajectory and some of the myths and confusion surrounding the subject. Hence the author introduces, contextualises and evaluates what is considered to be Spain's first institute of sociological research and study, the Instituto de Reformas Sociales (1903-1924). 